\title{
Creation of an evidence-based cookbook aimed at reducing the risk of dementia
}

\author{
M. P. Rayman, V. Ridland, K. Sharpe and P. V. Westcott \\ Faculty of Health and Medical Sciences, University of Surrey, Guildford, GU2 7 XH
}

Dementia, including its most common manifestation, Alzheimer's Disease (AD), is a major, and escalating, health problem worldwide. According to the Alzheimer's Society, it is the most feared disease among the over-55 s. Although media coverage reflects a strong public interest in dementia, it focuses mostly on personal stories, celebrities and 'miracle cures' rather than accurate, evidencebased advice or practical approaches to prevention.

In the absence of a cure, or even effective treatment for dementia, an increasing body of evidence suggests that modifying lifestyle factors, including diet, may help to reduce risk. The evidence, however, is not yet sufficient to make firm public-health recommendations leaving the way open for unscrupulous practitioners to cherry-pick and misrepresent otherwise valid research in order to pedal so-called 'superfoods' and supplements.

It is estimated that a 10-25\% reduction in seven risk factors (diabetes, mid-life hypertension, mid-life obesity, smoking, depression, low educational attainment, physical inactivity) - all with obvious links to diet - could potentially prevent $1-3$ million cases of dementia worldwide ${ }^{(1,2)}$. However most people are unaware that they can anything do to reduce risk.

Our aim was to produce an evidence-based cookbook to help people in midlife to make the most appropriate dietary choices to reduce their risk of dementia ${ }^{(3)}$. To this end we conducted an extensive review of the scientific literature on diet, nutrition, specific nutrients and supplements relevant to the incidence, progression and outcome of mild cognitive impairment (MCI), dementia and AD. An evaluation of the evidence concluded that there are few meaningful randomised controlled trials, mainly as a result of the long latency period that precedes the onset of symptoms. There is, however, a large body of observational evidence of varying quality. Our challenge was to adapt this for public consumption and to make the science meaningful to non-scientists.

The most robust evidence is for a range of foods and nutrients that benefit the brain, obtained from the diet rather than from supplements. These include fish, olive oil, citrus fruit, berries, pistachio nuts, beetroot, other fruits and vegetables, red wine and grape products, high-flavanol cocoa, vitamins $\mathrm{B}$ (particularly $\mathrm{B}_{12}$ and folate), $\mathrm{C}$ and $\mathrm{E}$, monounsaturated fatty acids and long-chain omega-3 fatty acids, consumed as part of an overall beneficial dietary pattern. There is increasing evidence of the role of the food matrix and food synergy (e.g. the Mediterranean diet) in the best outcomes ${ }^{(4)}$.

We used this evidence to choose the optimum ingredients and cooking methods for 100 recipes, selected and developed to reflect the overall dietary patterns identified as most likely to reduce dementia risk. We were able to put findings into context, making complex science engaging and readable. In doing this, we feel we have bridged the gap between academia and the popular press, providing realistic and honest advice. The practical and appealing recipe ideas make dietary adaptation an attractive, cost-effective and easy-to-implement option for the consumer. All recommended dietary interventions carry additional health benefits.

There is a need for further research to determine the long-term impact of this approach on the risk of dementia.

We gratefully acknowledge the support of the Waterloo Foundation for this project.

1. Barnes DE, Yaffe K. (2011) Lancet Neurol 10, 819-28.

2. Norton S, Matthews FE, Barnes DE et al. (2014) Lancet Neurol 13, 788-94.

3. Eskilinen M. Publications of the University of East Finland. Dissertations in Health Sciences 2014. Available at http://epublications.uef.fi/pub/ urn_isbn_978-952-61-1394-4/urn_isbn_978-952-61-1394-4

4. Jacobs DR Jr, Gross MD, Tapsell LC. (2009) AJCN 89, 1543S-1548S. 